\title{
A novel nonpharmacological intervention - breathing-controlled electrical stimulation for neuropathic pain management after spinal cord injury - a preliminary study
}

\author{
This article was published in the following Dove Press journal: \\ Journal of Pain Research \\ 3 November 2016 \\ Number of times this article has been viewed
}

\author{
Shengai $L i^{1,2}$ \\ Matthew Davis' \\ Joel E Frontera' \\ Sheng $\mathrm{Li}^{1,2}$ \\ 'Department of Physical Medicine and \\ Rehabilitation, University of Texas \\ Health Science Center at Houston, \\ ${ }^{2}$ TIRR Memorial Hermann Research \\ Center, TIRR Memorial Hermann \\ Hospital, Houston, TX, USA
}

Objective: The objective of this study was to examine the effectiveness of a novel nonpharmacological intervention - breathing-controlled electrical stimulation (BreEStim) - for neuropathic pain management in spinal cord injury (SCI) patients.

Subjects and methods: There were two experiments: 1) to compare the effectiveness between BreEStim and conventional electrical stimulation (EStim) in Experiment (Exp) 1 and 2) to examine the dose-response effect of BreEStim in Exp 2. In Exp 1, 13 SCI subjects (6 males and 7 females, history of SCI: 58.2 months, from 7 to 150 months, impairments ranging from C4 AIS B to L1 AIS B) received both BreEStim and EStim in a randomized order with at least 3 days apart. A total of 120 electrical stimuli to the median nerve transcutaneously were triggered by voluntary inhalation during BreEStim or were randomly delivered during EStim. In Exp 2, a subset of 7 subjects received BreEStim120 and 240 stimuli randomly on two different days with 7 days apart (BreEStim120 vs BreEStim240). The primary outcome variable was the visual analog scale (VAS) score.

Results: In Exp 1, both BreEStim and EStim showed significant analgesic effects. Reduction in VAS score was significantly greater after BreEStim $(2.6 \pm 0.3)$ than after EStim $(0.8 \pm 0.3)(P<0.001)$. The duration of analgesic effect was significantly longer after BreEStim (14.2 \pm 6 hours) than after EStim (1.9 \pm 1 hours) $(P=0.04)$. In Exp 2, BreEStim120 and BreEStim2 40 had similar degree and duration of analgesic effects.

Conclusion: The findings from this preliminary study suggest that BreEStim is an effective alternative nonpharmacological treatment for chronic neuropathic pain in patients suffering from SCI.

Keywords: neuromodulation, neuropathic pain, spinal cord injury, electrical stimulation, BreEStim

\section{Introduction}

Neuropathic pain is a chronic condition caused by a primary lesion or dysfunction of the nervous system. It is often described as burning, shooting, prickling, or electrical. The pain can be spontaneous, a painful response to innocuous stimuli (allodynia), or an exaggerated pain in response to noxious stimuli (hyperalgesia). ${ }^{1}$ Approximately $65 \%-85 \%$ of spinal cord injury (SCI) patients report experiencing neuropathic pain, and approximately one-third of them have severe neuropathic pain. ${ }^{2}$ Neuropathic pain does not resolve over time and in some cases worsens over time. ${ }^{3}$ Current pharmacological treatments provide incomplete and often insufficient relief. Pharmacological interventions
Correspondence: Sheng $\mathrm{Li}$ TIRR Memorial Hermann Research Center, TIRR Memorial Hermann Hospital, I 333 Moursund, Houston, TX 77030, USA

Tel +I 7137977I25

Email sheng.li@uth.tmc.edu 
are also associated with side effects, such as constipation and sedation. Therefore, neuropathic pain has increasingly been recognized as an important contributor to poorer physical, psychological, and social functioning in people with SCI. ${ }^{4-6}$ Alternative, nonpharmacological interventions for neuropathic pain management are in great need for this population.

Various neurostimulation techniques have been developed and used for neuropathic pain management after SCI, with varying degrees of success, ${ }^{7}$ including transcutaneous electrical nerve stimulation (TENS), ${ }^{8}$ electroacupuncture, ${ }^{9}$ spinal cord stimulation, ${ }^{10}$ deep brain stimulation, ${ }^{11}$ and transcranial direct current stimulation. ${ }^{12-14}$ Breathing-controlled electrical stimulation (BreEStim) is a recently developed, novel, nonpharmacological intervention for neuropathic pain management. ${ }^{15}$ Briefly, in the BreEStim treatment, single-pulse electrical stimulation (EStim) is delivered transcutaneously to a peripheral nerve. The delivery is triggered by fast and deep voluntary inhalation. In recent case reports, ${ }^{15,16}$ we have shown that BreEStim is effective in reducing chronic neuropathic pain in people with paraplegia and above-theknee amputation. In these cases, BreEStim was applied only to the median nerve in the arm. As such, it was not likely that BreEStim modified the source of pain at the periphery. These preliminary observations suggest that BreEStim could modify the affective component of neuropathic pain, ie, increased tolerance to pain. We further systematically compared the analgesic effects between BreEStim and conventional EStim to a peripheral nerve, or voluntary breathing only on experimentally induced pain in healthy subjects. We observed that BreEStim resulted in a general desensitization effect (measured by elevated electrical pain thresholds in both treated and nontreated hands), while there was no such effect, or even a general sensitization effect after EStim or voluntary breathing only. ${ }^{17-19}$ The findings support preliminary clinical observations $^{15,16}$ that BreEStim-induced analgesia is likely related to modification of the affective component, ie, central effect. This effect has been attributed to internal pain-coping mechanisms activated by EStim during the specific window of voluntary breathing. ${ }^{16}$

Mechanisms of neuropathic pain in SCI are not well understood. Pain is multidimensional, including sensory and affective (ie, unpleasantness) components. These components are processed in parallel and were thought to be inseparable. ${ }^{20,21}$ For example, when superficial tactile stimulation is applied to the hand area where acupuncture points are located, activation is seen only in somatosensory cortices. However, when the acupuncture points are stimulated, and pain is experienced by the subjects, activation of additional cortical areas, such as anterior cingulate cortex and insula, is observed. ${ }^{22}$ It is thus theoretically possible that the sensory and affective components of neuropathic pain could be modulated separately for therapeutic purposes. For example, BreEStim is shown to relieve pain by selectively affecting the affective component of neuropathic pain. ${ }^{15,16}$

The primary aim of this study was to examine the effectiveness of BreEStim for neuropathic pain management in people with SCI. Specifically, the analgesic effects of BreEStim and EStim for neuropathic pain were compared. Given the analgesic effect of BreEStim was observed in healthy subjects, ${ }^{17-19}$ it was hypothesized that BreEStim is able to produce greater analgesic effects than EStim in SCI subjects. We then explored the possible dose-response effect of BreEStim in a subset of SCI subjects. This information (degree and duration of analgesic effect) is important for future dosing studies.

\section{Subjects and methods Subjects}

Thirteen SCI subjects ( 6 males and 7 females and history of SCI: 58.2 months, from 7 to 150 months, impairments ranging from C4 AIS B to L1 AIS B) participated in this study. Detailed characteristics are listed in Table 1. Inclusion criteria were as follows: 1) neuropathic pain after SCI; 2) chronic pain lasting > 3 months; 3 ) between 18 and 75 years of age; and 4) stable oral pain medication regimen for at least 2 weeks. Patients were explicitly instructed to continue their pain medications regardless of experimental outcome, including the parameters for their intrathecal drug delivery pumps and spinal cord stimulation during the course of the study. Exclusion criteria were as follows: 1) currently adjusting pain medications for neuropathic pain; 2) pain which was not neuropathic or not related to SCI, eg, pain from carpal tunnel syndrome; 3 ) presence of a pacemaker; 4) inability to follow commands or to give consent; 5) presence of asthma or other pulmonary disease; and 6) medical instability. The pain syndromes were classified according to the International Spinal Cord Injury Pain Classification. ${ }^{23-25}$ To ensure that the pain was neuropathic in origin, only subjects with a Leeds assessment of neuropathic symptoms and signs (LANSS) score of $\geq 12$ were enrolled. ${ }^{26-28}$ The average LANSS score was 16.7, ranging from 12 to 23 (Table 1). All subjects gave written informed consent prior to participation. This study was approved by the Committee for the Protection of Human Subjects at the University of Texas Health Science Center at Houston and TIRR Memorial Hermann Hospital. 


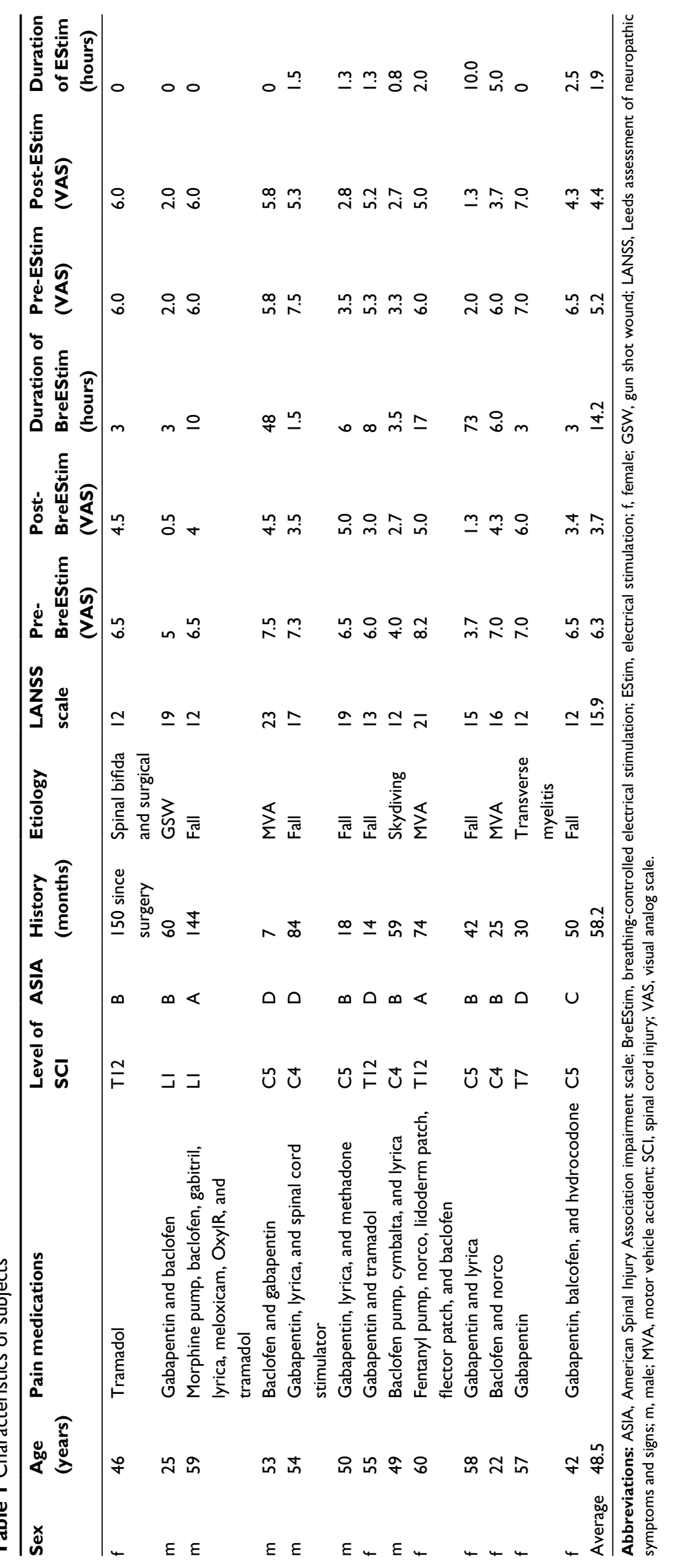




\section{Experimental procedures}

There were two experiments. In Experiment (Exp) 1, a total of 120 electrical stimuli during either BreEStim (BreEStim120) or EStim (EStim120) were delivered to all subjects in a randomized order with at least 3 days apart. In Exp 2, a subset of 7 subjects received an additional, higher dose of BreEStim (a total of 240 stimuli, BreEStim240) on a different day to explore the dose-response effect of BreEStim. Note that both BreEStim and EStim only performed once in each experiment.

In Exp 1, subjects were seated comfortably with two hands rested on the experimental table. A pair of trimmed surface electrodes $\sim 2 \times 2 \mathrm{~cm}^{2}$ was placed over the medial aspect of the distal forearm along the path of the median nerve from forearm to hand on the dominant hand side. Two electrodes were separated by $\sim 10 \mathrm{~mm}$. During BreEStim120, subjects wore a leak-proof face mask that was connected to a Pneumotach system (Hans Rodulph, Inc., Shawnee, KS, USA) to record the airflow rate. It was displayed on the computer screen to ensure sufficient inhalation effort. A single-pulse ( $0.1 \mathrm{~ms}$ square wave) EStim was delivered to the median nerve transcutaneously when the airflow rate reached the preset threshold level at $40 \%$ of its peak value. This usually occurred when subjects took a fast, strong, and deep inhalation. During EStim120, subjects wore the same face mask but were instructed to breathe normally. EStim pulses were randomly delivered every $1.5-2$ seconds. In both interventions, the same amount of EStim (120 stimuli) was delivered, and the intensity of EStim was controlled by subjects themselves. During both interventions, subjects were encouraged to gradually increase the intensity of EStim to a painful, yet tolerable level. This level was equivalent to $\sim 7-8$ on visual analog scale (VAS). Subjects were explicitly instructed that aversiveness of EStim was important for both BreEStim and EStim interventions. The intensity of EStim was recorded at the 1st, 20th, 40th, 60th, 80th, 100th, and 120th stimuli.

In Exp 2, a convenient subset of patient subjects (4 females and 3 males) was recruited to receive an additional session of BreEStim at a higher dose -240 stimuli (BreEStim240). BreEStim240 and BreEStim120 were given on 2 different days with 7 days between (BreEStim120 vs BreEStim240) in a randomized order. The same procedure was used for BreEStim 240 as in Exp 1.

Prior to each intervention in both experiments, subjects had a familiarization session to ensure that they understood the procedure and requirements. During each treatment session, breaks were encouraged and allowed upon request to ensure sufficient rest. All subjects tolerated treatment sessions well. No episodes of hyperventilation or hypoxia were reported.

These settings and protocols were similar to our recent series of BreEStim experiments. ${ }^{17-19}$ Technical details of BreEStim are available online in a methodology video article: http://www.jove.com/video/50077/. ${ }^{15}$

\section{Measurement}

The primary outcome measure was VAS score. VAS has been extensively used and validated. ${ }^{29}$ Specifically, VAS $(0-10$ point scale) score is recommended as an outcome measure for pain intensity after SCI. ${ }^{27}$ VAS score was assessed before and immediately after the intervention. Postintervention VAS score was also assessed through follow-up phone calls every 4 hours except nighttime (10 pm-7 am). It is important to emphasize that subjects were explicitly instructed to maintain the same pain medication regimen even if they felt that treatment had helped in pain reduction. The analgesic effect after BreEStim or EStim was based on subjective reports of the VAS score. The degree of analgesic effect, ie, reduction in VAS score or the difference between pre- and postintervention VAS scores was then calculated. The duration of analgesic effect was also recorded. It is known that SCI patients usually have different levels of pain at different locations of the body. ${ }^{30}$ To compare the overall analgesic effect between BreEStim and EStim, a global pain intensity was estimated from VAS scores averaged across different locations in this study. ${ }^{31}$

\section{Data analysis and statistics}

Two-way repeated measures ANOVAs with factors of INTERVENTION (pre vs post) and STIM (BreEStim120 vs EStim120, or BreEStim120 vs BreEStim240) were used to compare the analgesic effects using VAS scores. Two-way repeated measures ANOVAs were also used to compare the intensity of electrical current between two interventions (STIM) at different trials ( $\times 7$ levels, at 1st, 20th, 40th, 60th, 80th, 100th, and 120th). Paired $t$-tests were used to compare the duration and degree of analgesic effects between two interventions.

\section{Results}

In Exp 1, both BreEStim120 and EStim120 had significant analgesic effects. Averaged across 13 patients, VAS scores decreased from 6.3 to 3.7 after BreEStim 120 and from 5.2 to 4.4 after EStim120 (Figure 1). A repeated measures twoway ANOVA revealed a main effect of INTERVENTION $\left(F_{[1,12]}=31.38, P<0.001\right)$. No main effect of STIM was found. 

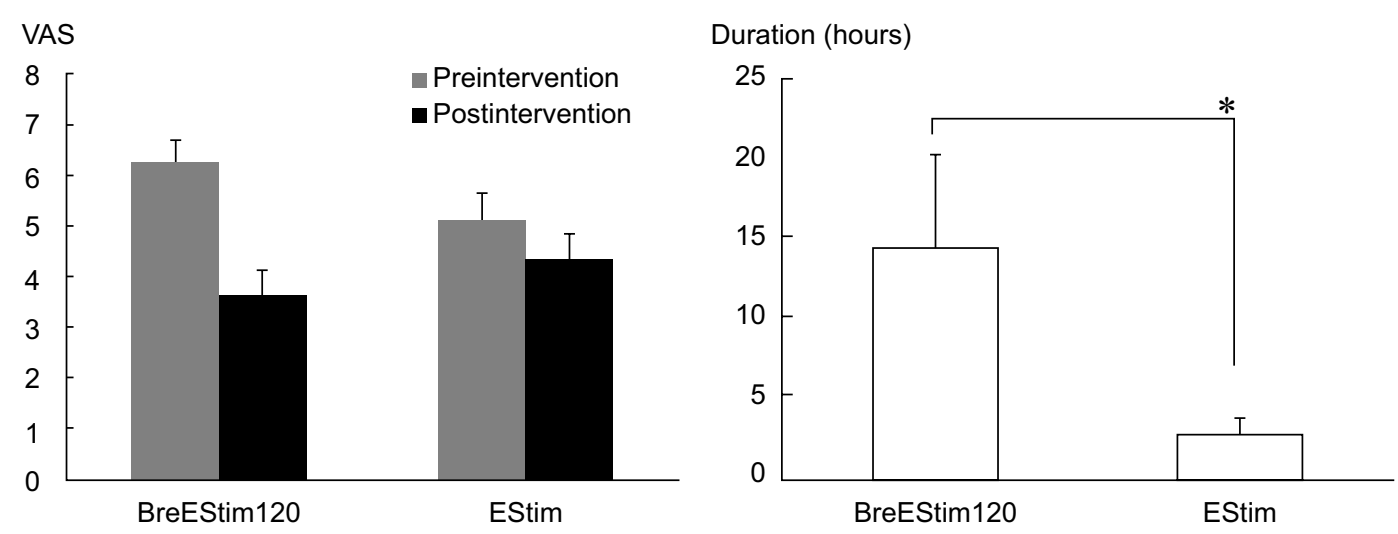

Figure I Comparisons of analgesic effects between BreEStim I20 and EStim.

Notes: Two-way repeated measures ANOVAs with factors of INTERVENTION (pre vs post) and STIM (BreEStim I 20 vs EStim I20) were used to compare the analgesic effects using VAS scores. Paired $t$-tests were used to compare the duration and degree of analgesic effects between two interventions. BreEStim 120 produced greater and longer analgesic effects than EStim I20. *indicates statistical significance.

Abbreviations: ANOVAs, analysis of variances; BreEStim, breathing-controlled electrical stimulation; EStim, electrical stimulation; VAS, visual analog scale.

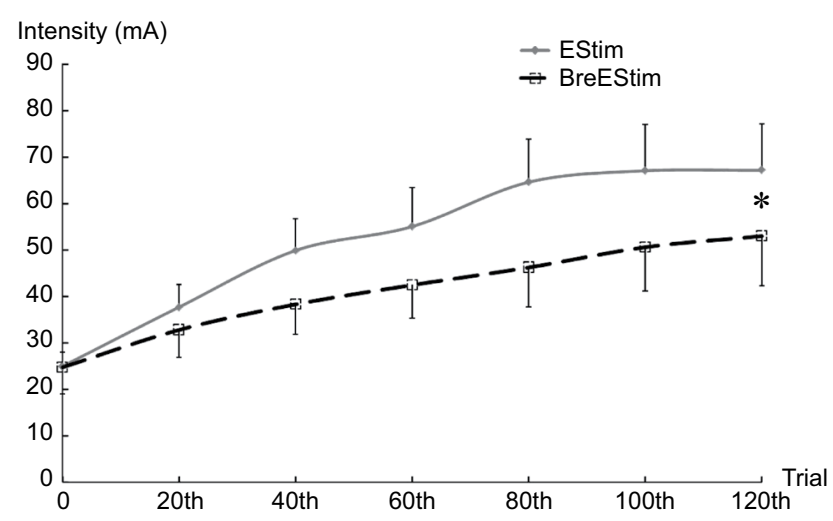

Figure 2 The intensity of electrical stimulation during BreEStim and EStim across the 120 trials.

Notes: Two-way repeated measures ANOVAs were also used to compare the intensity of electrical current between two interventions (STIM) at different trials ( $\times 7$ levels, at Ist, 20th, 40th, 60th, 80th, 100th, and 120th). The intensity of electrical stimulation was greater during EStim than during BreEStim. *indicates statistical significance.

Abbreviations: ANOVAs, analysis of variances; BreEStim, breathing-controlled electrical stimulation; EStim, electrical stimulation.

There was a significant INTERVENTION $\times$ STIM interaction $\left(F_{[1,12]}=27.19, P<0.001\right)$. Post hoc Turkey tests showed that pre-BreEStim120 VAS score (6.3 \pm 0.4$)$ was greater than all other VAS scores, post-BreEStim120 VAS score (3.7 \pm 0.5 ) was smaller than other VAS scores, and pre-EStim 120 VAS score (5.2 \pm 0.4$)$ was greater than post-EStim120 VAS score (4.4 \pm 0.5$)$. Reduction in VAS score was significantly greater

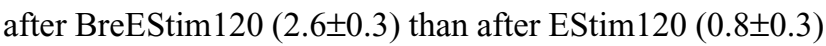
$(P<0.001)$. The duration of analgesic effect was significantly longer after BreEStim120 than after EStim120 $(P=0.04)$. On average, the analgesic effect lasted 14.2 \pm 6 hours after BreEStim120, but only $1.9 \pm 1$ hours after EStim120. As listed in Table 1, there was a contrasting analgesic effect for individual subjects. All subjects had some effects after BreEStim120, while 5 out of 13 subjects did not have any response after EStim120. The intensity of electrical current was significantly greater during EStim120 than during BreEStim $120\left(F_{[1,12]}=7.34, P=0.0189\right)$ (Figure 2 ).

In Exp 2, BreEStim120 and BreEStim240 had similar analgesic effects (Figure 3). On average, VAS score decreased from $6.8 \pm 0.50$ to $3.8 \pm 0.57$ after BreEStim120 and from $5.6 \pm 0.55$ to $3.4 \pm 0.41$ after BreEStim240. Repeated measures two-way ANOVAs showed a main effect of INTERVENTION $\left(F_{[1,6]}=62.84, P<0.001\right)$. There were no main effects of STIM or INTERVENTION $\times$ STIM interaction. Reduction of VAS score was also similar between BreEStim120 (2.7 \pm 0.59$)$ and BreEStim240 (2.9 \pm 0.29 ). Among the tested subjects, there were large variations in the duration of analgesic effects. On average, there was no significant difference in the duration between BreEStim120 (29.9 28.6 hours) and BreEStim 240

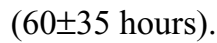

\section{Discussion}

In the present study, the main novel finding was that BreEStim120 produced greater and longer analgesic effects for SCI patients suffering from chronic neuropathic pain, as compared to EStim120. The intensity of EStim was greater in EStim120 than in BreEStim120. Furthermore, there was no dose-response effect between BreEStim120 and BreEStim240. This is the first study suggesting that BreEStiminduced analgesia observed in healthy subjects ${ }^{17-19}$ could be translated and applied to SCI patients. As mentioned earlier, pharmacological treatment is often inadequate and has considerable side effects. Patients with physical disabilities and pain, including SCI patients, have reported preference for nonpharmacological alternative treatments. ${ }^{32,33}$ These results are promising that BreEStim could serve as an alternative treatment for SCI patients for neuropathic pain management. 

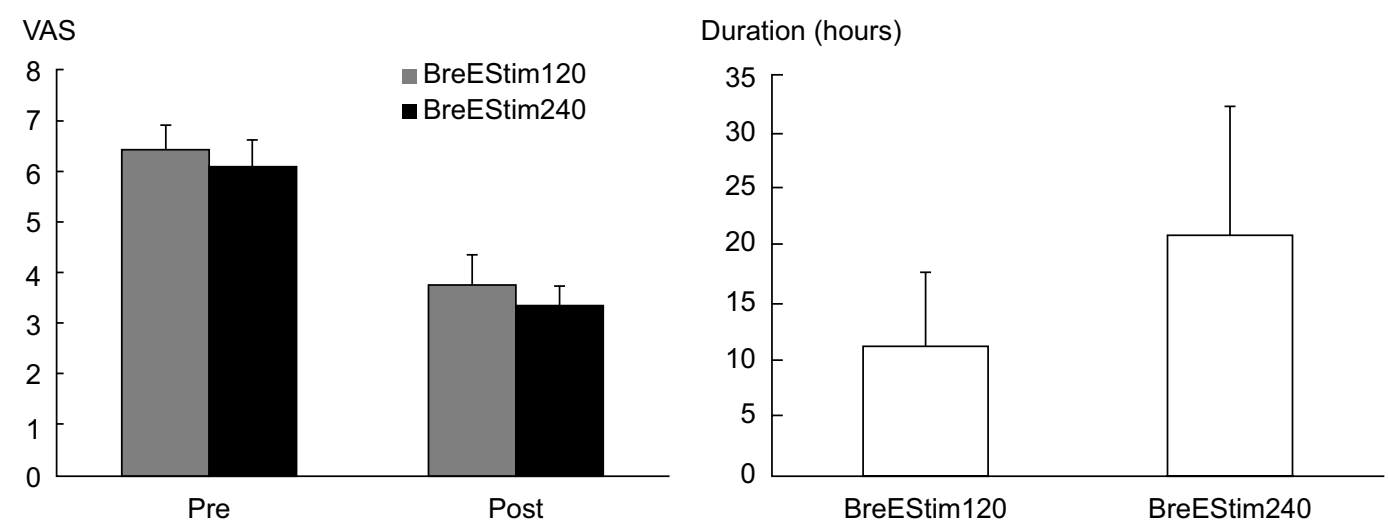

Figure 3 Comparisons of analgesic effects between BreEStim 120 and BreEStim240.

Notes: Two-way repeated measures ANOVAs with factors of INTERVENTION (pre vs post) and STIM (BreEStim I 20 vs BreEStim240) were used to compare the analgesic effects using VAS scores. Paired $t$-tests were used to compare the duration and degree of analgesic effects between two interventions. No dose-dependent analgesic effects were found between BreEStim 120 and BreEStim240.

Abbreviations: ANOVAs, analysis of variances; BreEStim, breathing-controlled electrical stimulation; VAS, visual analog scale.

EStim has been used for the treatment of pain after SCI. A Cochrane Review article showed variable results of TENS in the management of chronic pain, ${ }^{34}$ even when different parameters were tried, such as different frequencies of EStim in TENS. ${ }^{35}$ Similarly, variable responses to EStim were also observed in the present study. We found that 5 out 13 subjects did not respond to EStim. In contrast, all subjects had some analgesic effects after BreEStim. Overall, BreEStim had greater and longer analgesic effect than EStim in SCI with chronic neuropathic pain. This observation was consistent with previous findings of better analgesic effects of BreEStim on experimentally induced pain in healthy subjects. ${ }^{17-19} \mathrm{We}$ noticed that there was a significant difference in preintervention baseline pain levels. VAS scores were greater before BreEStim than before EStim. Post-BreEStim VAS scores were significantly smaller than post-EStim VAS scores. These results suggest that patients have had fluctuations in their baseline pain levels prior to the interventions, but BreEStim has had significant analgesic effects.

The findings of better analgesic effects after BreEStim than after EStim suggest that additional pain-coping mechanisms are likely integrated during BreEStim. In Exp 1, the subjects received the same amount of electrical stimuli (120 stimuli in total), but the intensity of EStim was greater during EStim than during BreEStim. We observed that 5 out of 13 patients did not benefit from EStim120. Though we cannot comment whether these patients would benefit from EStim with more stimulation (eg, 1,000 stimuli), EStim alone evidently did not play a critical role in analgesic effect during BreEStim. It has also been shown that EStim is reported to be effective in patients with peripheral neuropathic pain ${ }^{36}$ but less effective in patients with central neuropathic pain. ${ }^{37}$ Furthermore, pain-related brain activity was found to be reduced only during the fast inspiration period, as compared to other patterns of breathing. However, this decreased painrelated activity was dissociated from spinal nociceptive transmission. ${ }^{38}$ Taken together, our results suggest that central mechanisms play an important role in neuropathic pain in SCI patients. Possible central pain-coping mechanisms during BreEStim are related to unique features of voluntary breathing, as suggested in previous studies. ${ }^{17-19}$

\section{Limitations of the study}

There are limitations in this study. VAS score was the primary outcome variable. It is clinically meaningful and has been validated and recommended for SCI patients..$^{27,29}$ However, report of VAS score is subjective. It could be potentially biased by subjects, particularly in the follow-up assessment, even though we explicitly instructed subjects not to do so. In this study with a cross-over design, the bias of subjective reports is likely to be minimized by within-subject comparisons. Physiological biomarkers, such as heart rate variability (HRV), can be a valuable tool to assess analgesic effects objectively. ${ }^{39,40}$ HRV can be included as an objective outcome variable in future studies. Another limitation is small sample size. No significant difference in VAS score reduction and duration of analgesic effects was observed between BreEStim 120 and BreEStim 240 in the 7 subjects in Exp 2. In this preliminary study, large variations were observed. Alternatively, no difference in analgesic effects could be due to the fact that analgesic effects have reached the plateau with BreEStim120, and more stimulation would not make any further difference. We were not able to associate any particular components with a positive response or greater duration of effect, such as age, time since injury, level of injury, completeness or cause of injury, or presence of certain medication class (eg, opiates). 


\section{Conclusion}

These findings in this preliminary study suggest that BreEStim is an effective alternative nonpharmacological treatment for chronic neuropathic pain in patients suffering from SCI. BreEStim may be tried as a complement to the pharmacological treatment of neuropathic pain in SCI patients. Future studies with larger sample sizes may further support clinical application of BreEStim.

\section{Acknowledgments}

This study was supported in part by Mission Connect (013-109), TIRR Foundation, and NIH NICHD/NCMRR 1R21HD087128-01. The abstract of this article was presented at the 2015 AAP Annual Meeting, San Antonio, TX, USA, on March 10-14, and has also been published in the American Journal of Physical Medicine \& Rehabilitation, March 2015, Volume 94, Issue 3. SL holds US Patent number 8,229,566 "Method and Apparatus of Breathing-Controlled Electrical Stimulation for Skeletal Muscles", issued on July 24, 2012, and US Patent number 8,588,919 "Method and Apparatus of Breathing-Controlled Electrical Stimulation for Skeletal Muscles" Divisional of Application number 12/146,176 (issued as US Patent number 8,229,566). He was blinded to all experiments and did not have direct patient contact in this study.

\section{Disclosure}

The authors report no conflicts of interest in this work.

\section{References}

1. Bennett MI. Neuropathic Pain. New York: Oxford University Press Inc; 2010.

2. Siddall PJ, McClelland JM, Rutkowski SB, Cousins MJ. A longitudinal study of the prevalence and characteristics of pain in the first 5 years following spinal cord injury. Pain. 2003;103(3):249-257.

3. Jensen MP, Kuehn CM, Amtmann D, Cardenas DD. Symptom burden in persons with spinal cord injury. Arch Phys Med Rehabil. 2007;88(5):638-645.

4. Norrbrink Budh C, Hultling C, Lundeberg T. Quality of sleep in individuals with spinal cord injury: a comparison between patients with and without pain. Spinal Cord. 2005;43(2):85-95.

5. Stormer S, Gerner HJ, Gruninger W, et al. Chronic pain/dysaesthesiae in spinal cord injury patients: results of a multicentre study. Spinal Cord. 1997;35(7):446-455.

6. Jensen MP, Chodroff MJ, Dworkin RH. The impact of neuropathic pain on health-related quality of life: review and implications. Neurology. 2007;68(15):1178-1182.

7. Kotze A, Simpson KH. Stimulation-produced analgesia: acupuncture, TENS and related techniques. Anaesth Intensive Care Med. 2008;9(1):29.

8. Norrbrink Budh C, Lundeberg T. Non-pharmacological pain-relieving therapies in individuals with spinal cord injury: a patient perspective. Complement Ther Med. 2004;12(4):189-197.

9. Ulett GA, Han S, Han JS. Electroacupuncture: mechanisms and clinical application. Biol Psychiatry. 1998;44(2):129.
10. Finnerup NB, Yezierski RP, Sang CN, et al. Treatment of spinal cord injury pain. Pain Clin Updates. 2001;9:1-6.

11. Murphy D, Reid DB. Pain treatment satisfaction in spinal cord injury. Spinal Cord. 2001;39(1):44-46.

12. Fregni F, Boggio PS, Lima MC, et al. A sham-controlled, phase II trial of transcranial direct current stimulation for the treatment of central pain in traumatic spinal cord injury. Pain. 2006;122(1-2): 197-209.

13. Boggio PS, Zaghi S, Fregni F. Modulation of emotions associated with images of human pain using anodal transcranial direct current stimulation (tDCS). Neuropsychologia. 2009;47(1):212-217.

14. Boggio PS, Zaghi S, Lopes M, Fregni F. Modulatory effects of anodal transcranial direct current stimulation on perception and pain thresholds in healthy volunteers. Eur J Neurol. 2008;15(10):1124-1130.

15. Li S. Breathing-controlled electrical stimulation (BreEStim) for management of neuropathic pain and spasticity. J Vis Exp. 2013; 10(71):e50077.

16. Li S, Melton DH, Berliner JC. Breathing-controlled electrical stimulation (BreEStim) could modify the affective component of neuropathic pain after amputation: a case report. J Pain Res. 2012;5:71-75.

17. Li S, Berliner JC, Melton DH, Li S. Modification of electrical pain threshold by voluntary breathing-controlled electrical stimulation (BreEStim) in healthy subjects. PLoS One. 2013;8(7):e70282.

18. Li S, Hu T, Beran MA, et al. Habituation to experimentally induced electrical pain during voluntary-breathing controlled electrical stimulation (BreEStim). PLoS One. 2014;9(8):e104729.

19. $\mathrm{Hu} \mathrm{H}, \mathrm{Li} \mathrm{S}, \mathrm{Li}$ S. Pain modulation effect of breathing-controlled electrical stimulation (BreEStim) is not likely to be mediated by fast and deep voluntary breathing. Sci Rep. 2015;5:14228.

20. Price DD. Psychological and neural mechanisms of the affective dimension of pain. Science. 2000;288(5472):1769-1772.

21. Frot M, Mauguière F, Magnin M, Garcia-Larrea L. Parallel processing of nociceptive A-delta inputs in SII and midcingulate cortex in humans. J Neurosci. 2008;28(4):944-952.

22. Hui KKS, Liu J, Makris N, et al. Acupuncture modulates the limbic system and subcortical gray structures of the human brain: evidence from fMRI studies in normal subjects. Hum Brain Mapp. 2000;9(1):13.

23. Bryce TN, Biering-Sorensen F, Finnerup NB, et al. International spinal cord injury pain (ISCIP) Classification: part 2. Initial validation using vignettes. Spinal Cord. 2012;50(6):404-412.

24. Bryce TN, Biering-Sorensen F, Finnerup NB, et al. International spinal cord injury pain classification: part I. Background and description. March 6-7, 2009. Spinal Cord. 2012;50(6):413-417.

25. Mahnig S, Landmann G, Stockinger L, Opsommer E. Pain assessment according to the international spinal cord injury pain classification in patients with spinal cord injury referred to a multidisciplinary pain center. Spinal Cord. Epub 2016 Jan 12.

26. Bennett M. The LANSS pain scale: the leeds assessment of neuropathic symptoms and signs. Pain. 2001;92(1-2):147-157.

27. Bryce TN, Budh CN, Cardenas DD, et al. Pain after spinal cord injury: an evidence-based review for clinical practice and research - report of the National Institute on Disability and Rehabilitation Research Spinal Cord Injury Measures Meeting. J Spinal Cord Med. 2007;30(5): 421-440.

28. Hallström H, Norrbrink C. Screening tools for neuropathic pain: can they be of use in individuals with spinal cord injury? Pain. 2011;152(4):772-779.

29. McCarthy M Jr, Chang CH, Pickard AS, et al. Visual analog scales for assessing surgical pain. J Am Coll Surg. 2005;201(2):245-252.

30. Miró J, Gertz KJ, Carter GT, Jensen MP. Pain location and functioning in persons with spinal cord injury. PM R. 2014;6(8):690-697.

31. Raichle KA, Osborne TL, Jensen MP, Cardenas D. The reliability and validity of pain interference measures in persons with spinal cord injury. J Pain. 2006;7(3):179-186.

32. Krauss HH, Godfrey C, Kirk J, Eisenberg DM. Alternative health care: its use by individuals with physical disabilities. Arch Phys Med Rehabil. 1998;79(11):1440-1447. 
33. Haythornthwaite JA, Wegener S, Benrud-Larson L, et al. Factors associated with willingness to try different pain treatments for pain after a spinal cord injury. Clin J Pain. 2003;19(1):31-38.

34. Nnoaham KE, Kumbang J. Transcutaneous electrical nerve stimulation (TENS) for chronic pain. Cochrane Database Syst Rev. 2008;16(3):CD003222.

35. Norrbrink C. Transcutaneous electrical nerve stimulation for treatment of spinal cord injury neuropathic pain. J Rehabil Res Dev. 2009;46(1):89-94.

36. Cruccu G, Aziz TZ, Garcia-Larrea L, et al. EFNS guidelines on neurostimulation therapy for neuropathic pain. Eur J Neurol. 2007;14(9): 952-970.
37. Meyler WJ, de Jongste MJ, Rolf CA. Clinical evaluation of pain treatment with electrostimulation: a study on TENS in patients with different pain syndromes. Clin J Pain. 1994;10(1):22-27.

38. Arsenault M, Ladouceur A, Lehmann A, Rainville P, Piché M. Pain modulation induced by respiration: phase and frequency effects. $\mathrm{Neu}$ roscience. 2013;252:501-511.

39. Koenig J, Jarczok MN, Ellis RJ, Hillecke TK, Thayer JF. Heart rate variability and experimentally induced pain in healthy adults: a systematic review. Eur J Pain. 2014;18(3):301-314.

40. Chalaye P, Goffaux P, Lafrenaye S, Marchand S. Respiratory effects on experimental heat pain and cardiac activity. Pain Med. 2009;10(8): 1334-1340.

\section{Publish your work in this journal}

The Journal of Pain Research is an international, peer reviewed, open access, online journal that welcomes laboratory and clinical findings in the fields of pain research and the prevention and management of pain. Original research, reviews, symposium reports, hypothesis formation and commentaries are all considered for publication.

\section{Dovepress}

The manuscript management system is completely online and includes a very quick and fair peer-review system, which is all easy to use. Visit http://www.dovepress.com/testimonials.php to read real quotes from published authors. 